\title{
Development of immunoglobulin $G$ enzyme-linked immunosorbent assay for the serodiagnosis of severe acute respiratory syndrome
}

\author{
Pei-Lan Shao ${ }^{1}$, Po-Ren Hsueh ${ }^{2}$, Luan-Yin Chang ${ }^{1}$, Chun-Yi Lu ${ }^{1}$, Chuan-Liang Kao ${ }^{2}$, \\ Yu-Ping Chiang ${ }^{1}$, Hsiang-Yi Huang ${ }^{1}$, Fu-Yuan Huang ${ }^{3}$, Chin-Yun Lee ${ }^{1}$, Lung-Ji \\ Chang ${ }^{4}$, T.-C. $\mathrm{Wu}^{5}$, Li-Min Huang ${ }^{1, *}$, and the SARS Research Group of National \\ Taiwan University College of Medicine and National Taiwan University Hospital \\ ${ }^{1}$ Department of Pediatrics and ${ }^{2}$ Department of Laboratory Medicine, National Taiwan University Hospital, \\ Taipei, Taiwan; ${ }^{3}$ Department of Pediatrics, Taipei Mackay Memorial Hospital Taipei, Taiwan; ${ }^{4}$ Department \\ of Molecular Genetics and Microbiology, University of Florida, Florida, USA; ${ }^{5}$ Departments of Pathology, \\ Oncology, Obstetrics and Gynecology and Molecular Microbiology and Immunology, The Johns Hopkins \\ University, Maryland, USA
}

Received 17 June 2004; accepted in revised form 25 August 2004

(C) 2005 National Science Council, Taipei

Key words: coronavirus, enzyme-linked immunosorbent assay, serodiagnosis, severe acute respiratory syndrome

\section{Summary}

Severe acute respiratory syndrome (SARS) is an emerging infectious disease caused by a novel SARSassociated coronavirus (SARS-CoV). The clinical characteristics are high fever, rapidly progressive diffuse pneumonitis and respiratory distress. It is highly infectious through intimate contact or direct contact with infectious body fluids. Outbreaks within communities and hospitals have been reported. Development of rapid and reliable diagnostic tools is urgently needed. We developed an immunoglobulin G (IgG) enzyme-linked immunosorbent assay (ELISA), using whole virus antigen of SARS-CoV. Eighty-six serum samples collected from patients who were hospitalized for other causes were examined to determine the cut-off O.D. value. The cut-off O.D. value was defined as 0.175 by calculating the mean O.D. value of the 86 sera plus 3 standard deviations. To determine the sensitivity and specificity of the ELISA, 56 positive sera and 204 negative sera were tested. The sensitivity was $96.4 \%$ and the specificity was $100 \%$. The results suggest that the IgG ELISA using whole virus antigen of SARS-CoV has a high sensitivity and specificity in detecting SARS IgG antibodies. This IgG ELISA is a powerful tool for serodiagnosis of SARS.

\section{Introduction}

Severe acute respiratory syndrome (SARS) is an emerging infectious disease. The clinical characteristics are fever, dry cough, myalgia, dyspnea, lymphopenia, and abnormal chest radiograph

*To whom correspondence should be addressed. Fax: + 886-22393-4749; E-mail: lmhuang@ha.mc.ntu.edu.tw results [1,2]. A significant proportion of patients with SARS have a fulminant course characterized by high fever, rapidly progressive diffuse pneumonitis and respiratory failure [3]. Cases of SARS were first reported in Guandong Province of southern China in November 2002, followed by reports from Vietnam, Hong Kong, Singapore, Canada, and Taiwan $[1,2,4,5]$. As of 29 September 2003, there have been 8098 persons reported as infected and 774 patients have died because of 
SARS. The case fatality ratio has reached $9.6 \%$ [5].

The responsible pathogen for SARS is a newly discovered SARS-associated coronavirus (SARS$\mathrm{CoV})[6,7]$. This virus is transmitted through contact with respiratory droplets, fomites, or respiratory secretions. The virus is highly contagious and able to cause outbreaks within communities and hospitals $[1,2,4]$. Delayed diagnosis and failure to isolate SARS patients in hospitals can lead to the transmission of this virus to healthcare workers and other patients $[1,4,8]$. However, it is very difficult to distinguish SARS-CoV infection from other acute viral respiratory diseases, such as influenza, based on the clinical presentations [8,9]. Current laboratory diagnosis of SARS$\mathrm{CoV}$ infection is achieved by isolating the virus by cell culture or detecting the viral nucleic acids by reverse-transcriptase polymerase chain reaction (RT-PCR). These methods are either time-consuming or of inadequate sensitivities. For that reason, developing a rapid and reliable diagnostic tool is of urgent need. In this study, we describe a sensitive and specific ELISA in detecting SARS$\mathrm{CoV} \operatorname{IgG}$ for use in routine diagnosis and for large-scale population surveillance.

\section{Materials and methods}

\section{Preparation and identification of whole-virus} antigen for ELISA

SARS-CoV isolate (TW1) [4] was propagated in Vero E6 cells, in MEM medium (JRH, Lenexa, USA) supplemented with $2 \%$ fetal calf serum (FCS). Viruses were harvested from culture supernatant at $48 \mathrm{~h}$ after inoculation when more than $90 \%$ of cells showed cytopathogenic effects. After centrifugation $(600 \times g$ for $10 \mathrm{~min})$ and discarding the pellets containing exfoliated cells, the supernatants were heated at $56{ }^{\circ} \mathrm{C}$ for $1 \mathrm{~h}$ to inactivate SARS-CoV and were filtered through $0.2 \mu \mathrm{M}$ pores (Sartorius, Goettingen, Germany) to dispose of bacteria. The supernatants were suspended in $20 \%$ polyethylene glycol (Sigma, St. Louis, MO, USA) and centrifuged again at $8000 \times g$ for $4 \mathrm{~h}$ at $4{ }^{\circ} \mathrm{C}$. The pellets containing viruses were resuspended in PBS buffer and purified by $35-80 \%$ sucrose gradients centrifugation $\left(8000 \times g\right.$ for $16 \mathrm{~h}$ at $\left.4{ }^{\circ} \mathrm{C}\right)$. The viral fraction was washed with PBS and then centrifuged at $8000 \times g$ for $4 \mathrm{~h}$ at $4{ }^{\circ} \mathrm{C}$. The pellets contained the purified whole cell viral antigens. The concentration of purified viral proteins was measured using a Bio-Rad protein assay kit (Bio-Rad, Hercules, CA, USA).

The purified whole virus antigen was subjected to polyacrylamide electrophoresis and stained with Coomassie blue and Western blotting analysis using SARS patients' sera to understand the proteins contained in this preparation.

\section{IgG ELISA using SARS-CoV whole virus antigen}

Purified viral protein $(5 \mu \mathrm{g})$ was diluted in $200 \mu \mathrm{l}$ $50 \mathrm{mM} \mathrm{Na}_{2} \mathrm{CO}_{3}(\mathrm{pH} 9.5$ ) and was used to coat the wells of microtiter plates at $4{ }^{\circ} \mathrm{C}$ overnight. The plates were then blocked with $380 \mu \mathrm{l} /$ well blocking buffer [5\% FCS in Superblock (PIERCE, Rockford, IL, USA)] at room temperature for $1 \mathrm{~h}$. The sera were pre-treated at $56{ }^{\circ} \mathrm{C}$ for $30 \mathrm{~min}$ and then diluted at 1:50 in blocking buffer. Two hundred microliters of diluted sera were added to each well and incubated at room temperature for $30 \mathrm{~min}$. The plates were then washed five times in washing buffer containing PBS and $0.5 \%$ Tween 20. Thereafter, $200 \mu$ l of goat anti-human IgG antibody (Chemicon, Temecula, CA, USA) diluted at 1: 80,000 in blocking buffer was added to each well and incubated at room temperature for $30 \mathrm{~min}$. The plates were washed five times in washing buffer again. The specific anti-SARS-CoV IgG was detected by adding $100 \mu \mathrm{l}$ of TMB (KPL, Gaithersburg, MD, USA). Absorbance was measured at $450 \mathrm{~nm}$ after adding $100 \mu \mathrm{l}$ of $\mathrm{H}_{2} \mathrm{SO}_{4}$ solution (1N).

\section{Cut-off value determination}

We tested sera collected from patients visiting National Taiwan University Hospital for diseases other than SARS to determine the cut-off O.D. value for the IgG ELISA. None of the patients whose sera were used for cut-off value determination had a prior contact history with SARS patients. All the sera had been confirmed to be seronegative for SARS-CoV by immunofluorescence assay (IFA) at the dilution of 1:25. The mean O.D. value of the sera plus 3 standard deviations was calculated as the cut-off O.D. value of the IgG ELISA. 


\section{ELISA diagnostic sensitivity and specificity}

To determine the sensitivity and specificity of the ELISA, true positive and true negative control sera were defined and tested. True positive control sera were those collected from patients of SARS and tested positive by IFA. Clinical SARS patients were diagnosed according to WHO criteria [10]. True negative control sera were those collected in 2000 and 2001 before SARS had been first reported and tested negative by IFA.

\section{Statistical analysis}

Data were analyzed with the SPSS Statistical Package (Version 11.5, Leadtools, Chicago, IL, USA). Spearman rank order correlation was used

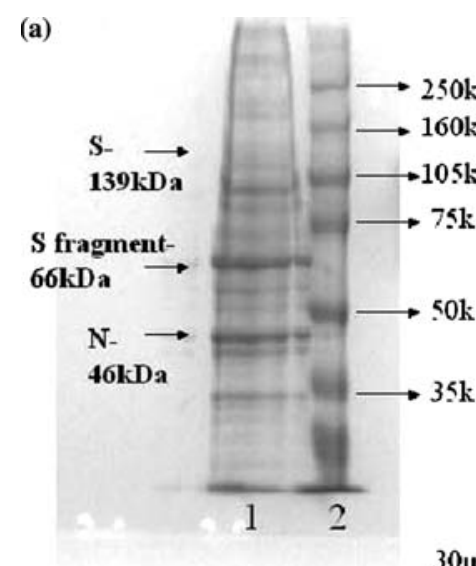

$7.5 \%$ SDS PAGE

\section{$30 \mu \mathrm{g} /$ lane}

to examine the correlation between O.D. values and time of patients' sera collection.

\section{Results}

\section{Constituents of purified virus antigen}

The purified SARS CoV antigen underwent an SDS PAGE and was stained with Coomassie blue as well as patients' sera (Figure 1). Several antigens were identified by the Coomassie blue stain in our whole virus preparation, including the four structural proteins, including spike (S), envelope $(\mathrm{E})$, membrane $(\mathrm{M})$, and nucleocapsid $(\mathrm{N})$ [11]. From the results of Western blotting, the major antigen recognized by the patients' sera was the $46 \mathrm{kDa} \mathrm{N}$ protein.

(b)

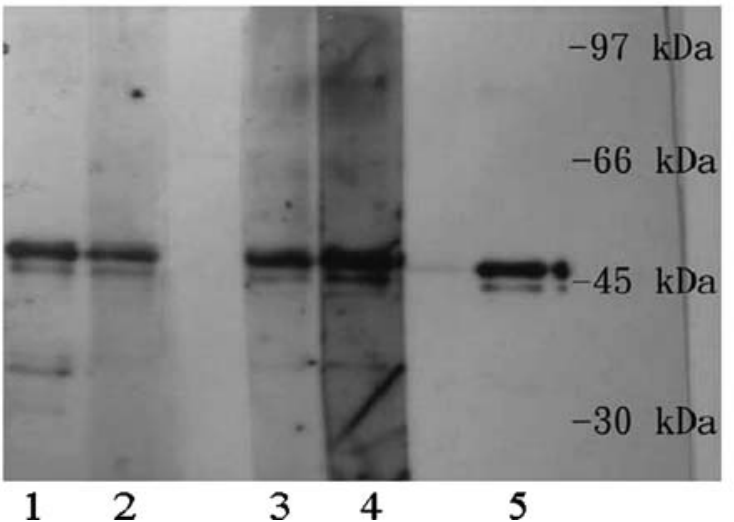

Figure 1. SDS-polyacrylamide gel electrophoresis (SDS-PAGE) analysis and Western blot analysis of SARS-CoV whole virus antigen preparations. (a) Purified whole virus antigens were subjected to electrophoresis on 7.5 and 10\% SDS-PAGE gels (lane 1). Proteins were visualized by staining with Coomassie Blue. Bands corresponding to S, S fragment, N, M, and E proteins are identified. The molecular masses of protein standard (lane 2) are shown at the right. (b) Western blot analysis of SARS-CoV whole virus antigen preparations using four different patients' sera is shown in lane 1 to lane 4 . There is a major antigen recognized by patients' sera. The size of the major antigen is compatible with the mouse monoclonal anti- $\mathrm{N}$ antibody (lane 5). 


\section{Cut-off value determination}

Eighty-six sera collected from patients visiting National Taiwan University Hospital for other causes were tested for cut-off value determination, including 33 with respiratory tract infections, 30 with non-respiratory infections, and 23 with noninfectious diseases. All the sera were seronegative when tested by IFA. The mean O.D. value and the standard deviation were calculated to be 0.064 and 0.037 , respectively. Thus, the cut-off value was defined to be 0.175 from the 86 sera.

\section{ELISA diagnostic sensitivity and specificity}

\section{Sensitivity}

Fifty-six serum samples collected serially from 18 SARS patients were tested by both IFA and ELISA. There were three males and 15 females. Their ages ranged from 11 to 70 years old. All 18 patients had fever and pneumonia during April and May of 2003. Among them, eight were healthcare workers of two hospitals that had nosocomial SARS outbreaks [12]. Six had visited or had been admitted to those two hospitals during the outbreak period. Three had household contact with a SARS patient. Detection of SARS-CoV nucleic acid by RT-PCR in plasma, throat swab, or stool samples was done in 11 patients, and 5 patients had positive results.

All the sera were positive for IgG anti-SARS $\mathrm{CoV}$ using IFA. They were collected from 3 to 110 days (median, 17.5 days) after fever onset. Fifty-four of the 56 sera were reactive in the $\mathrm{IgG}$ ELISA (O.D. value higher than the defined cut-off of 0.175 ). The O.D. value of the 56 positive sera ranged from 0.115 to 0.686 , and the mean O.D. value was 0.286 (Figure 2). Forty-nine (87.5\%) sera had an O.D. value higher than 0.2. The two false negative sera were collected 10 and 12 days after fever onset, respectively. The five sera that had O.D. values between 0.175 and 0.2 were collected 4, 10, 13, 14 and 20 days after onset of fever, respectively. The O.D. values tended to be higher, while the sera were collected later in the disease course $(p<0.001)$ [Figure 3]. The sensitivity of the SARS-CoV IgG ELISA was $96.4 \%$.

\section{Specificity}

Two hundred and four sera collected in 2000 and 2001 before SARS had been first reported were

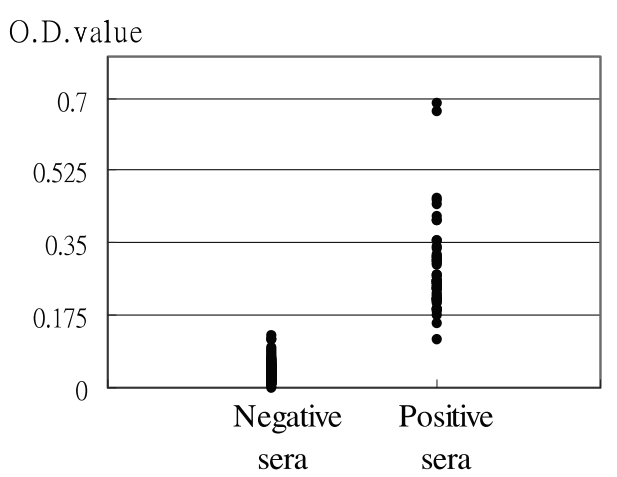

Figure 2. Scatter chart of 204 negative control sera and 56 positive control sera tested by the SARS-CoV IgG ELISA. The cut-off O.D. value was defined as 0.175 .

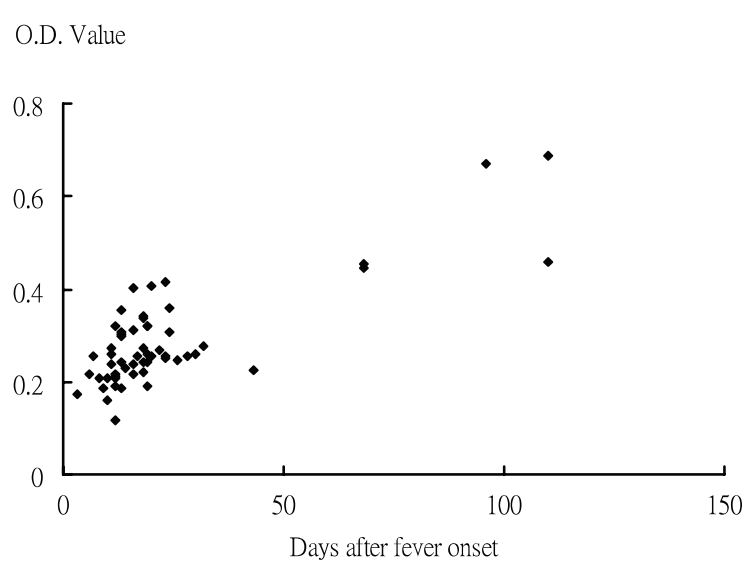

Figure 3. Correlation between the O.D. values and the time of patients' sera collection. The Spearman coefficient was 0.586 and $p$-value $<0.001(N=56)$.

considered as true negative controls. These sera were collected from healthy children and teenagers of different age groups: 57 from children aged less than 2 years old, 47 from 2 to 5 years old, 50 from 6 to 10 years old, and 50 from 11 to 15 years old. None of these sera had $\operatorname{IgG}$ antibody against SARS-CoV when tested by IFA. All 204 sera were seronegative by the ELISA with a mean O.D. of 0.036 (Figure 2). Two hundred and one sera $(98.5 \%)$ had O.D. values less than 0.1 . The specificity of the SARS-CoV IgG ELISA was $100 \%$.

\section{Discussion}

In this study, we developed a SARS-CoV IgG ELISA using whole SARS-CoV antigen. This IgG 
ELISA is able to differentiate between positive and negative sera. The mean O.D. values for the positive sera and negative sera were 0.286 and 0.036 , respectively. With the cut-off O.D. value set at 0.175 , all 204 negative control sera collected in 2000 and 2001 had O.D. values lower than the cutoff, while 54 of the 56 positive controls collected from SARS patients had O.D. values higher than the cut-off. The sensitivity of the SARS-CoV IgG ELISA using whole SARS-CoV antigen is $96.4 \%$, and the specificity is $100 \%$. The positive predictive value is $100 \%$ and the negative predictive value is $99 \%$. The results also correlate well with the IFA results.

There are two key steps in developing a good ELISA to detect antibodies against viral antigens. The first step is defining an appropriate cut-off value for positive readings, while the second step involves reducing the background noise. We set the cut-off value at the mean O.D. value plus 3 standard deviations after testing 86 negative serum samples. With the derived cut-off value (0.175), we were able to accurately differentiate seropositive from seronegative serum samples with high sensitivity $(96.4 \%)$ and specificity $(100 \%)$.

Initially we used skim milk-based blocking buffer as suggested by several protocols [13,14]. However, the background O.D. values were unacceptably high (data not shown). Therefore, we tried a new blocking buffer containing fetal calf serum and Superblock. We also increased the concentration of Tween 20 to $0.5 \%$ in the washing buffer. These modifications successfully reduced the non-specific reaction in the SARS-CoV IgG ELISA. Fetal calf serum has been used in the blocking buffer in some protocols $[15,16]$. One previous study also showed that blocking buffers containing fetal calf serum are able to reduce the background noise that is present in protocols using skim milk-based blocking buffers [16].

In this study, the whole SARS-CoV (TW1) was used as the antigen for the specific ELISA. Compared with recombinant viral protein as antigen, the advantages in using whole virus antigen are speed and simplicity of preparation without the need for recombinant-DNA techniques and expression, and purification of viral proteins. Recombinant protein antigen-based ELISA was found to be of low specificity in one ELISA for enterovirus 71 antibody detection [14]. Furthermore, infected patients usually mount variable antibody responses to several antigens of a virus [17]. Using multiple virus antigens theoretically could be more sensitive in determining antibody response compared with assays using single viral antigen.

Safety issues are always a concern when developing a whole virus-based ELISA. In addition to operating in a level 3 biosafety laboratory, this protocol used prolonged heat of $60 \mathrm{~min}$ to inactivate the virus. This is necessary because there is evidence suggesting that heating less than $60 \mathrm{~min}$ is ineffective in sterilizing SARS CoV (S.R. Shih, personal communication).

Most positive sera in this study had O.D. values higher than 0.2. In the 7 sera with O.D. values lower than 0.2 (including two false negative sera), six were collected no later than 14 days after fever onset. Thus, we believe that a low O.D. value for a serum sample obtained within 2 weeks of fever onset should be interpreted with caution. Serial sampling is recommended to establish the diagnosis.

The 204 negative control sera were collected from children and teenagers under 16 years old during 2000 and 2001. These subjects were young and were supposedly experiencing frequent virus infections. None of these sera were reactive. Thus, it is suggested that cross-reactions to other prevalent viruses among children and teenagers is not a major concern in the SARS-CoV IgG ELISA.

In conclusion, we successfully developed an IgG ELISA using whole SARS-CoV antigen. The protocol is quick and simple, with high sensitivity and specificity in detecting SARS IgG antibodies. The IgG ELISA is a powerful tool for rapid serodiagnosis of SARS and for seroepidemiology survey.

\section{Acknowledgements}

This work was conducted by the SARS Research Group of National Taiwan University College of Medicine and National Taiwan University Hospital. This group consists of Ding-Shinn Chen, Ming-Fu Chang, Shan-Chwen Chang, Bor-Luen Chiang, Horn-Nerg Ho, Jin-Town Wang, WeiKung Wang, and Pan-Chyr Yang. This work was supported by the National Research Program for Genomic Medicine grant NSC92-2751-B-002-013$\mathrm{Y}$ from the National Science Council, the Executive Yuan, Taiwan. 


\section{References}

1. Lee N., Hui D., Wu A., Chan P., Cameron P., Joynt G.M., Ahuja A., Yung M.Y., Leung C.B., To K.F., Lui S.F., Szeto C.C., Chung S. and Sung J.J.Y., A major outbreak of severe acute respiratory syndrome in Hong Kong. New Engl. J. Med. 348: 1986-1994, 2003.

2. Poutanen S.M., Low D.E., Henry B., Finkelstein S., Rose D., Green K., Tellier R., Draker R., Adachi D., Ayers M., Chan A.K., Skowronski D.M., Salit I., Simor A.E., Slutsky A.S., Doyle P.W., Krajden M., Petric M., Brunham R.C. and McGeer A.J., The National Microbiology Laboratory, Canada and the Canadian Severe Acute Respiratory Syndrome Study Team. Identification of severe acute respiratory syndrome in Canada. New Engl. J. Med. 348: 1995-2005, 2003.

3. Peiris J.S.M., Chu C.M., Cheng V.C.C., Chan K.S., Hung I.F.N., Poon L.L.M., Law K.I., Tang B.S.F., Hon T.Y.W., Chan C.S., Chan K.H., Ng J.S.C., Zheng B.J., Ng W.L., Lai R.W.M., Guan Y., Yuen K.Y. and The HKU/UCH SARS Study Group. Clinical progression and viral load in a community outbreak of coronavirusassociated SARS pneumonia: a prospective study. Lancet 361: 1767-1772, 2003.

4. Hsueh P.R., Hsiao C.H., Yeh S.H., Wang W.K., Chen P.J., Wang J.T., Chang S.C., Kao C.L., Yang P.C. and The SARS Research Group of National Taiwan University College of Medicine and National Taiwan University Hospital. Microbiologic characteristics, serologic responses, and clinical manifestations in severe acute respiratory syndrome, Taiwan. Emerg. Infect. Dis. 9: 11631167, 2003.

5. World Health Organization, 2003. Summary of probable SARS cases with onset of illness from 1 November 2002 to 31 July 2003. [Online] http: //www.who.int/sars/country/ table2003_09_23/en/. Accessed 26 September 2003.

6. Drosten $\bar{C}$., Gunther S., Preiser W., Werf S.V.D., Brodt H.R., Becker S., Rabenau H., Panning M., Kolesnikova L., Fouchier R.A.M., Berger A., Burguiere A.M., Cinatl J., Eickmann M., Escriou N., Grywna K., Kramme S., Manuguerra J.C., Muller S., Rickerts V., Sturmer M., Vieth S., Klenk H.D., Osterhaus A.D.M.E., Schmitz H. and Doerr H.W., Identification of a novel coronavirus in patients with severe acute respiratory syndrome. New Engl. J. Med. 348: 1967-1976, 2003.

7. Ksiazek T.G., Erdman D., Goldsmith C.S., Zaki S.R Peret T., Emery S., Tong S., Urbani C., Comer J.A., Lim W, Rollin P.E., Dowell S.F., Ling A.E., Humphrey C.D., Shieh W.J., Guarner J., Paddock C.D., Rota P., Fields B., DeRisi J., Yang J.Y., Cox N., Hughes J.M., LeDuc J.W., Bellini W.J., Anderson L.J. and The SARS Working Group. A novel coronavirus associated with severe acute respiratory syndrome. New Engl. J. Med. 348: 1953-1966, 2003.

8. Booth C.M., Matukas L.M., Tomlinson G.A., Rachlis A.R., Rose D.B., Dwosh H.A., Walmsley S.L., Mazzulli T., Avendano M., Derkach P., Ephtimios I.E., Kitai I., Mederski B.D., Shadowitz S.B., Gold W.L., Hawryluck L.A., Chenkin J.S., Cescon D.W., Poutanen S.M. and Detsky A.S., Clinical features and short-term outcomes of 144 patients with SARS in the greater Toronto area. J. Am. Med. Assoc. 289: 2801-2809, 2003.

9. Hon K.L.E., Leung C.W., Cheng W.T.F., Chan P.K.S., Chu W.C.W., Kwan Y.W., Li A.M., Fong N.C., Ng P.C., Chiu M.C., Li C.K., Tam J.S. and Fok T.F., Clinical presentations and outcome of severe acute respiratory syndrome in children. Lancet 361: 1701-1703, 2003.

10. World Health Organization, 2003. Case definitions for surveillance of severe acute respiratory syndrome (SARS). [Online] http: //www.who.int/csr/sars/casedefinition/en/. Accessed 1 May 2003.

11. Lai M.M.C. SARS virus: the beginning of the unraveling of a new coronavirus. J. Biomed. Sci. 10: 664-675, 2003.

12. Centers for Disease Control and Prevention. Severe acute respiratory syndrome - Taiwan, 2003. MMWR Morb. Mortal. Wkly. Rep. 52: 461-466, 2003.

13. Saijo M., Qing T., Niikura M., Maeda A., Ikegami T., Prehaud C., Kurane I. and Morikawa S., Recombinant nucleoprotein based enzyme-linked immunosorbent assay for detection of immunoglobulin $\mathrm{G}$ to Crimean Congo hemorrhagic fever virus. J. Clin. Microbiol. 40: 15871591, 2002.

14. Tsao K.C., Chan E.C., Chang L.Y., Chan P.Y., Huang C.G., Chen Y.P., Chang S.C., Lin T.Y., Sun C.F. and Shih S.R., Reponses of IgM for enterovirus 71 infection. J. Med. Virol. 68: 574-580, 2002.

15. Bacon R.M., Biggerstaff B.J., Schriefer M.E., Gilmore R.D., Philipp M.T., Steere A.C., Wormser G.P., Marques A.R. and Johnson B.J., Serodiagnosis of Lyme disease by kinetic enzyme-linked immunosorbent assay using recombinant VlsE1 or peptide antigens of Borelia burgdorferi compared with 2-tierd testing using whole cell lysates. J. Infect. Dis. 187: 1187-1199, 2003.

16. Qing T., Saijo M., Lei H., Niikura M., Maeda A., Ikegami T., Xinjung W., Kurane I. and Morikawa S., Detection of immunoglobulin $\mathrm{G}$ to Crimean-Congo hemorrhagic fever virus in sheep sera by recombinant nucleoprotein-based enzyme-linked immunosorbent and immunofluorescence assays. J. Virol. Meth. 108: 111-116, 2003.

17. Robert T.S., Epstein-Barr virus (infectious mononucleosis). In: Mandell G.L., Bennett J.E. and Dolin R. (Eds), Principles and Practice of Infectious Diseases, 5th edn. Churchill Livingstone, Philadelphia, 2000, pp. 1599-1613. 